\title{
REVIEW PAPER ON SEGMENTATION METHODS FOR MULTIOBJECT FEATURE EXTRACTION
}

\author{
Sonali Deshmukh ${ }^{1}$, Anjali Yadav ${ }^{2}$ \\ ${ }^{1} M E$ (II ${ }^{\text {nd }}$ year), Department of ENTC, Smt Kashibai Navle College of Engineering, Maharashtra, India \\ ${ }^{2}$ Asst. Prof., Department of ENTC, Smt Kashibai Navle College of Engineering, Maharashtra, India
}

\begin{abstract}
Feature extraction and representation plays a vital role in multimedia processing. It is still a challenge in computer vision system to extract ideal features that represents intrinsic characteristics of an image. Multiobject feature extraction system means a system that can extract features and locations of multiple objects in an image. In this paper we have discuss various methods to extract location and features of multiple objects and describe a system that can extract locations and features of multiple objects in an image by implementing an algorithm as hardware logic on a field-programmable gate array-based platform. There are many multiobject extraction methods which can be use for image segmentation based on motion, color intensity and texture. By calculating zeroth and first order moments of objects it is possible to obtain locations and sizes of multiple objects in an image.
\end{abstract}

Keywords: multiobject extraction, image segmentation

\section{INTRODUCTION}

In recent years computer programs are become popular for processing digitize pictorial information.This area of digital pictorial information processing involved performing "local" tasks on picture "neighbourhoods."As researchers have shown,many of picture processing transformations that can be achieved by applying certain tasks independently or simultaneously to each element of the given image. Many multiobject extraction methods which can be use for image segmentation are based on motion, color intensity and texture. In Image segmentation image is divide into regions or categories, corresponding to the different objects or parts of objects. Image segmentation methods such as watershed transformation, graph-cut methods, clustering methods and neural network approaches have many recent developments. Most of these methods are suitable for complicated scenes in many cases such as heart surgery, brain surgery or multimedia. This paper focus on modification of algorithms use in tracking multiple targets with imaging sensors used onboard an air airborne platform.

\section{LITERATURE SURVEY}

\subsection{Watershed based Image Segmentation}

Paper [3] study the watersheds in edge-weighted graphs and define the watershed cuts following the intuitive idea. of drops of water flowing on a topographic surface. Watershed cuts is used as a fundamental method in many powerful segmentation procedures. The watershed cut correspond to idea of drops of water on a topographic surface flows towards the "nearest" minimum. the uniformity of watersheds can be defined by catch basins. Then by applying an equivalence theorem, their optimality can be proved in terms of spanning forests. After this, linear time algorithm is use to compute them. This is the most efficient algorithm in theory as well as in practice. If we consider gray scale image as a topographic surface the pixel gray level becomes the elevation of a point, the deeper parts like valley and the basins are correspond to dark areas of the topographic surface, whereas the crest lines and higher points correspond to the light areas. In watershed method set of points are divide such that they satisfied "drop of water principle".

In first step the uniformity of watershed cuts is establish, by proving that they can be equivalently defined by their catchment basins or by separating lines through the drop of water principle. Second step establishes the optimality of watershed-cuts. the link between minimum spanning forests and flooding from marker algorithms is shown in [19], by F. Meyer. Third step consists of a linear-time algorithm which computes the watershed-cuts of an edge weighted graph

\subsection{Grab-Cut based Image Segmentation}

For grab cut method the paper [5], focus on a new technique for general purpose interactive segmentation of $\mathrm{N}$ dimensional images in which certain pixels are marked as "object" or "background" to provide hard constraints for segmentation whereas soft constraints include both boundary and region information. The globally optimal segmentation of the $\mathrm{N}$-dimensional image is found by grab cuts. The obtained solution satisfying the constraints which gives the best balance of boundary and region properties among all segmentations. The topology of our segmentation consists of several isolated parts for both "object" and "background" segments. This paper presents some experimental results in the context of medical image segmentation and photo/video editing. In segmentation process "object" or "background provides clues on what the user intends to segment and then computing a global optimum among all segmentations for rest of the image. 
Boundary and region properties of the segments are defined as cost function. These properties can be considered as soft constraints for segmentation. User can add or remove any hard constraints and can recomputed efficiently using globally optimal segmentation. This helps to get any desired segmentation results quickly via very intuitive interaction.

\subsection{Clustering based Image Segmentation}

In [6] author present an implementation of Lloyd's k-means clustering algorithm, which is the filtering algorithm. This algorithm requires a kd-tree as the only major data structure. It is easy for implementation. The practical efficiency of the filtering algorithm is establish in two ways. Firstly by presenting a data-sensitive analysis of the algorithm's running time, this shows that the algorithm runs faster as the separation between clusters increases. Secondly it focus on a number of empirical studies both on real data sets from applications in color quantization, data compression, image segmentation and on synthetically generated data. The simplest method of image segmentation is thresholding technique. This technique is based on a clip-level (or a threshold value) to turn a gray-scale image into a binary image. The key of this technique is to select the threshold value (or values when multiple-levels are selected). Several popular methods are used in industry including the maximum. entropy method, Otsu's method (maximum variance), and k-means clustering.

\subsection{Artificial Neural Network based Segmentation}

Paper [7] describes artificial neural network. It is often called a neural network which is an artificial representation of human brain that tries to simulate its learning process. It is widely use in medical image segmentation. Neural network constitutes a large number of parallel nodes and Each of them can perform some basic computing. It is based on life simulation, especially the human brain's learning process. By transferring the connections among nodes and connection weights the learning process can be achieved. During the image segmentation process neural network can also reduce the requirements of expert intervention.

Table 1: Comparision of different segmentation methods

\begin{tabular}{|c|c|c|c|}
\hline $\begin{array}{l}\text { Segmentat } \\
\text { ion } \\
\text { technique }\end{array}$ & Methodology & Advantages & $\begin{array}{l}\text { Disadvantag } \\
\text { es }\end{array}$ \\
\hline $\begin{array}{l}\text { Watershed } \\
\text { Transfor } \\
\text { Mation }\end{array}$ & 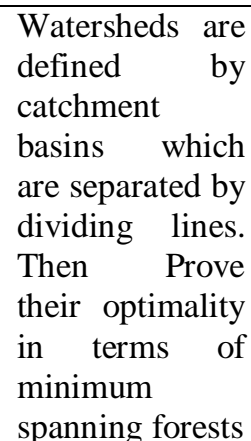 & $\begin{array}{l}\text { The } \\
\text { proposed } \\
\text { algorithm is } \\
\text { most } \\
\text { efficient in } \\
\text { both } \\
\text { theory and } \\
\text { in } \\
\text { practice }\end{array}$ & $\begin{array}{l}\text { - } \quad \text { Mo } \\
\text { re sensitive } \\
\text { to noise } \\
\text { suitable for } \\
\text { High } \\
\text { Resolution }\end{array}$ \\
\hline $\begin{array}{l}\text { Graph } \\
\text {-Cut }\end{array}$ & $\begin{array}{l}\text { Here } \\
\text { detailed }\end{array}$ & \begin{tabular}{|l|} 
This \\
technique
\end{tabular} & $\begin{array}{l}\text { multiway } \\
\text { cut problem }\end{array}$ \\
\hline
\end{tabular}

\begin{tabular}{|c|c|c|c|}
\hline & $\begin{array}{l}\text { technical } \\
\text { description of } \\
\text { basic } \\
\text { combinatorial } \\
\text { optimization } \\
\text { frame work for } \\
\text { segmentation } \\
\text { via s/t graphs }\end{array}$ & $\begin{array}{l}\text { has } \\
\text { great } \\
\text { potential for } \\
\text { solving } \\
\text { problems } \\
\text { in } \\
\text { vision and } \\
\text { graphics }\end{array}$ & $\begin{array}{l}\text { is hard and } \\
\text { thus } \\
\text { guaranteed } \\
\text { global } \\
\text { optimality } \\
\text { can be loose }\end{array}$ \\
\hline Clustering & $\begin{array}{l}\mathrm{K} \text {-means } \\
\text { clustering } \\
\text { method is used }\end{array}$ & $\begin{array}{l}\text { Efficiency is } \\
\text { increase } \\
\text { because data } \\
\text { points do } \\
\text { not } \\
\text { vary } \\
\text { through- } \\
\text { out } \\
\text { the } \\
\text { computa- } \\
\text { tion }\end{array}$ & $\begin{array}{l}\text { Algorithm } \\
\text { used is } \\
\text { quite } \\
\text { complex } \\
\text { and slow in } \\
\text { practice }\end{array}$ \\
\hline $\begin{array}{l}\text { Neural } \\
\text { Network } \\
\text { Approache } \\
\text { s }\end{array}$ & $\begin{array}{l}\text { Neural } \\
\text { networks are } \\
\text { use to perform } \\
\text { classification or } \\
\text { clustering }\end{array}$ & $\begin{array}{l}\text { No need to } \\
\text { write } \\
\text { complicated } \\
\text { programs }\end{array}$ & $\begin{array}{l}- \text { It } \\
\text { requires } \\
\text { Long } \\
\text { training time } \\
\text { - Res } \\
\text { ult may be } \\
\text { affected by } \\
\text { initialization }\end{array}$ \\
\hline
\end{tabular}

\section{PROPOSED SYSTEM}

Most of the video cameras used in general systems are restricted to some specific video formats. These formats are suitable for characteristics based on human eye which means the system processing speed is limited to recognition speed of human eye. This system describes a model that can extract locations and features of multiple objects in an image at high frame rate.

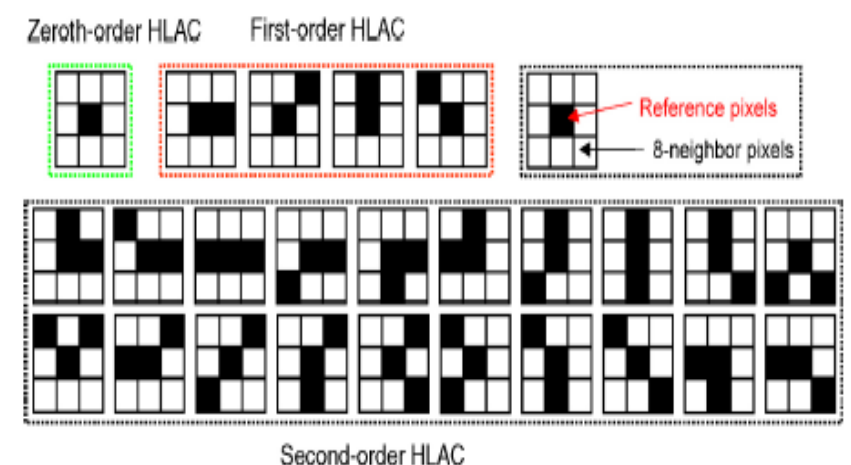

Fig 1.Twenty-five local patterns for HLACs

Here a cell-based multiobject feature extraction algorithm is implemented on hardware. Hardware consists of 25 higherorder local autocorrelation HLAC feature of 1024 objects in an image that can be simultaneously extracted for multiobject recognition. This algorithm can reduce memory consumption and computational complexity in labeling 
process for multiobject extraction by exchanging its computational sequence in labeling process of divided cells

\subsection{Implementation}

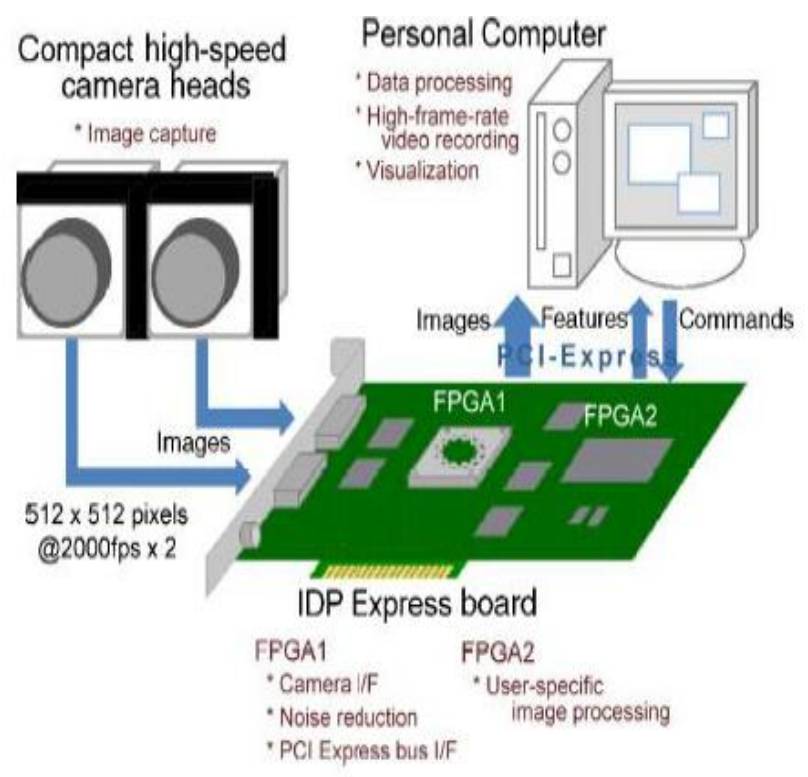

Fig 2. Configuration of High Speed vision: IDP Express

For camera head, FASTCAM MH4-10K (Photron) is used as input. There are two FPGA's in hardware, configuration programmable read-only memories for the FPGAs, serial-toparallel converters for camera inputs, and first-in first-out (FIFO) memories for data transfer between the FPGAs on a IDP express board. Here, 8-bit color images can be captured on its image sensor by using a Bayer color filter which were transferred at $2000 \mathrm{f} / \mathrm{s}$ for $512 \times 512$ pixels with digital serial communication in parallel with six pixels FPGA 1 is use to control camera I/O and PCI-e bus. FPGA 2 is used for hardware implementation of user-specified tasks. PCI-E bus is use to connect IDP express board to PC. 8-bit $512 \times 512$ images can be transferred at $4000 \mathrm{f} / \mathrm{s}$ for two camera heads [1].

\subsection{Hardware implementation logic}

The hardware implementation logic of multiobject feature extraction can be divided into several modules which includes binarization module , a cell-based labeling module , a cell-based features calculation module, and a data selector module for FIFO output. Figure above shows the schematic data flow of the implemented circuit.

The binarization module perform the function of converting $512 \times 512$ input images into binary images by scanning from the upper left to the lower right in units of eight pixels using $X$ and $Y$ address signals. This module can convert both color and gray images into binary images in parallel for 8 pixels. For color image a color conversion submodule converts RGB images into 8-bit HSV color images , hue $H(\boldsymbol{r})$, saturation $S(\boldsymbol{r})$, and value $V(\boldsymbol{r})$, in parallel with 8-pixel data after RGB conversion. After this HSV images are converted which are obtained after calculating the cells moments of $n \times n$ pixels based on the additivity in calculation of moment. to a binary image $B(\boldsymbol{r})$ by specifying a certain vivid and bright color with four thresholds of $\theta_{H L}, \theta_{H H}, \theta_{S}, \theta_{V}$.

When $\theta_{H L}<\theta_{H H}$

$B(r)= \begin{cases}1, & \left(H \in\left[\theta_{H L}, \theta_{H H}\right], S \geq \theta_{S}, V \geq \theta_{V}\right. \\ 0, & \text { (otherwise) }\end{cases}$

Otherwise

$B(r)= \begin{cases}1, & \left(H \notin\left[\theta_{H H}, \theta_{H L}\right], S \geq \theta_{S}, V \geq \theta_{V}\right) \\ 0, & \text { (otherwise) }\end{cases}$

For Gray image sensor, an input image $\mathrm{I}(\mathrm{r})$ is converted to binary image $\mathrm{B}(\mathrm{r})$ with threshold $\theta_{L}$ is given below

$$
I(r)= \begin{cases}1, & \left(\theta_{L} \leq I(r)\right) \\ 0, & (\text { otherwise })\end{cases}
$$

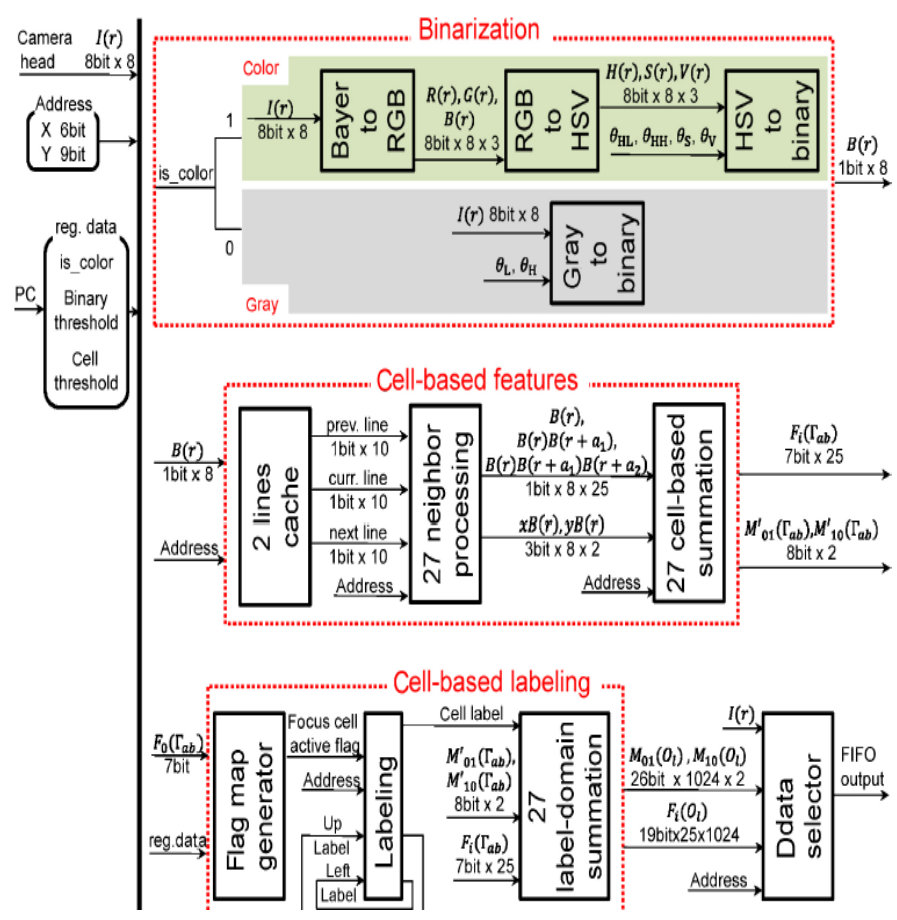

Fig. 3. Schematic data flow for implemented circuit

The cell based feature extraction module performs calculation of 25 HLACS , Fi $(\Gamma a b)(i=0, \ldots, 24)$, and two first moments, $M 10(\Gamma a b)$ and $M 01(\Gamma a b)$, for 4096 cells $\Gamma a b$ of $8 \times 8$ pixels by using a two-line cache, 27 neighborprocessing submodules, and 27 cell-based summation submodules. Zerothorder moment can be calculated by sharing the circuit for the zerothorder HLAC in this module.

Cell-Level HLAC Calculation:

For every cell $\Gamma_{\mathrm{ab}}$ of $\mathrm{n} \times \mathrm{n}$ pixels 25 HLACs $F_{i}\left(\Gamma_{a b}\right)$ are calculated as follows.

$F_{i}\left(\Gamma_{a b}\right)=\sum_{r \in \Gamma_{a b}} B(r) B\left(r+a_{1}\right) B\left(r+a_{2}\right)$ 
The cell-based labeling module can use a flag map submodule, a labeling submodule, and 27 label-domain summation submodules to calculate 25 HLACs and two first-order moments, for 1024 labeled regions .

The data selector module select FIFO output for an external PC with $X$ and $Y$ address signals from the input image or the 27 types of label-domain features of 1024 labeled objects. This all circuit module can be implemented on FPGA 2 of the IDP Express board

\section{CONCLUSION}

In this review paper of feature extraction, the overview of various segmentation methodologies applied for digital image processing is explained. It also describe the proposed system based on cell-based multiobject feature extraction Algorithm which is implemented on hardware and can extract sizes, positions, and HLACs of 1024 labeled objects in an image by dividing a $512 \times 512$ image into $64 \times 64$ cells, and it can also perform multiobject extraction of $512 \times 512$ images in real time at $2000 \mathrm{f} / \mathrm{s}$.

\section{REFERENCES}

[1] Qingyi Gu, Takeshi Takaki, and Idaku Ishii, Fast FPGA-Based Multiobject Feature Extraction," IEEE Transactions On Circuits And Systems For Video Technology, Vol. 23, No. 1, January 2013.

[2] G. Hamarneh and X. Li, "Watershed segmentation using prior shape and appearance knowledge," Image Vis. Comput., vol. 27, no. 1, pp. 59-68,2009

[3] J. Cousty, G. Bertrand, L. Najman, and M. Couprie, "Watershed cuts: Minimum spanning forests and the drop of water principle," IEEE Trans. Patt. Anal. Mach. Intell., vol. 31, no. 8, pp. 1362-1374, Aug. 2009.

[4] Rother, V. Kolmogorov, and A. Blake, "GrabCut: Interactive foreground extraction using iterated graph cuts," presented at the ACM SIGGRAPH, New York, 2004.

[5] Y. Boykov and G. Funka-Lea, "Graph cuts and efficient N-D image segmentation," Int. J. Comput. Vis., vol. 70, no. 2, pp. 109-131, 2006.

[6] T. Kanungo, D. M. Mount, N. S. Netanyahu, C. D. Piatko, R. Silverman, and A. Y. Wu, "An efficient k-means clustering algorithm: Analysis and implementation," IEEE Trans. Patt. Anal. Mach. Intell., vol. 24, no. 7 pp. 881-892, Jul. 2002.

[7] H.P. Narkhede Review of Image Segmentation Techniques International Journal of Science and Modern Engineering (IJISME) ISSN: 2319-6386, Volume-1, Issue-8, July 2013

[8] Y. Boykov and M.-P. Jolly, "Interactive graph cuts for optimal boundary and region segmentation of objects in N-D images," in Proc. Int. Comput. Vis., 2001, pp. 105-112.

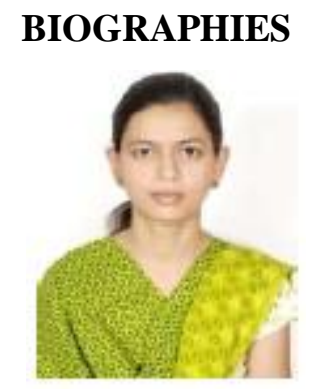

Ms. Sonali P. Deshmukh, received the Diploma in Engineering and B.E. degrees in Electronics and Telecommunication Engineering from Sant Gadge Baba Amravti University in 2009 and 2012, respectively. Currently pursuing M.E in VLSI \& Embedded Systems degree from SKNCOE, Pune under Savitribai Phule Pune University

Mrs. Anjali A. Yadav, currently assistant Professor in Department of Electronics and Telecommunication Engineering ,SKNCOE, Pune under Savitribai Phule Pune University 